\title{
Current Trends in Irish Perinatal Mortality
}

R Mahony, ME Foley, C 'OHerlihy

National Maternity Hospital, Holles St, Dublin 2

\section{Abstract}

This was a retrospective review of normally formed perinatal deaths among 176,620 births at the National Maternity Hospital (1984-2007). Prelabor stillbirths were categorised by presumed cause of death including unexplained, intrauterine growth retardation (IUGR), placental abruption, red cell alloimmunisation (RCA) and deaths related to prematurity. Peripartum deaths included intrapartum and first week neonatal deaths. The post-mortem rate, initially almost $100 \%$, fell to $60 \%$. Data were analysed using the Mantel-Haenszel chi square test for trends. In the study period there was a significant reduction in the PNM, largely because of a fall in death related to prematurity, term peripartum death, death at 42 weeks or greater, placental abruption, death related to IUGR and RCA (P<0.01). Overall the unexplained still birth rate was unchanged throughout the study period ( $\mathrm{p}=0.8)$ despite a highly significant $(p<0.001)$ increase in obstetric intervention particularly induction of labor and caesarean section.

\section{Introduction}

Many Countries in the developed world have seen a decline in perinatal mortality over the past few decades. ${ }^{1}$ During this time there have been many changes in obstetric practice including the introduction of routine obstetric ultrasound, intrapartum fetal monitoring and major advances in neonatal intensive care. One of the most obvious changes has been the rise in obstetric interventions with some units reporting up to a fivefold increase in the rate of caesarean section. Despite this, late pregnancy continues to be a time of relatively high mortality, with particular concern focusing on the persistence of late fetal deaths occurring after 28 weeks gestation. ' it is estimated that stillbirth (SB) accounts for $60 \%$ of all perinatal deaths and $75 \%$ of all preventable $10 s s e s$ (perinatal death of a normally formed infant weighing $1000 \mathrm{~g}$ or more). In the UK one in 200 infants is stillborn with a recent rise in non-anomalous SB rate noted. It has been reporgted that unexplained antepartum SB is the largest single contributor to perinatal mortality in the Western world. It is important to continually examine trends in perinatal mortality and to identify the reasons for these deaths if we are to develop strategies to further reduce perinatal mortality. The aim of our study was to examine trends among the principle categories of normally formed singleton perinatal deaths in Ireland over the past two decades.

\section{Methods}

Over the study period all perinatal deaths were discussed at a monthly multidisciplinary meeting which included obstetric, paediatric and pathology specialists and each case was recorded in the Annual Hospital Report which provided the data for this paper. Perinatal deaths due to lethal congenital malformation were excluded and the perinatal mortality rate was calculated per thousand births greater than $500 \mathrm{~g}$ and also against the denominator of ongoing pregnancies where appropriate. Unexplained stillbirths, deaths attributed to intrauterine growth retardation and placental abruption were analyzed separately for term pregnancies and for those less than 37 completed weeks gestation- preterm. Peripartum deaths intrapartum deaths and first week neonatal deaths were reported for term pregnancies only. Deaths related to prematurity (but excluding prelabor intrauterine death) and multiple pregnancies pregnancies only. Deaths related to prematurity (but excluding prelabor intrauterine death) and multiple pregnancies were reported separately. In recent years there has been a tendency to attribute some unexplained term stillbirths to
fetomaternal haemorrhage, sub clinical placental separation and cord accidents e.g. true knots, tight nucal cord and where these are identified they have been included in the overall analyses of term unexplained stillbirths. The where these are identified they have been included in the overall analyses of term unexplained stillbirths. The square test for trends.

\section{Results}

Among 176,620 non-anomalous births the corrected perinatal mortality fell from 10.6 in 1984 to $7.4 / 1000$ in 2007 $(\mathrm{p}<0.001)$. The caesarean section rate increased from $4.2 \%$ to $18.9 \%$ and the induction rate increased from $10.8 \%$ to $24.8 \%(\mathrm{p}<0.001)$.

Figure 1: Overall perinatal mortality/1000 deliveries, caesarean section rates and induction of labour rates (per cent) over 24 years at NMH

Term unexplained still births and term deaths attributed to IUGR

The PMR among term still births was unchanged - average $1.2 / 1000$ over the study period ( $P=0.8$ ) and was the same when calculated for all births or for ongoing pregnancies ( $>37$ weeks). In one third of cases (and increasingly so in recent years) a possible/ probably explanation for unexplained stillbirth has been identified - fetomaternal haemorrhage, sub clinical placental separation and cord accidents e.g. true knots, tight nucal cord. There was no significant change in the PMR attributed to IUGR (>37weeks), average 0.4/1000. (Trend; $p=0.6$ )

Peripartum deaths

There was a highly significant downward trend among term intrapartum and neonatal deaths (peripartum deaths) attributed to intrapartum asphyxia $(p<0.001)$.

Deaths at 42 weeks or greater

The overall incidence of pregnancies recorded as 42 weeks or greater fell from $12 \%$ to $6 \%$ and there was a significant fall in PNM in this cohort. When PMR was calculated to include unexplained still births and peripartum deaths among ongoing pregnancies greater than 42 weeks, the PNM averaged 5/1000 in the early years but with only one death recorded in the past 4 years $(p<0.001)$.

Figure 2: Trends for PNM among unexplained stillbirths $>37$ weeks gestation $(\mathrm{P}=0.8)$ and where a possible sentinel event (cord, silent placental abruption, feto-maternal haemorrhage) may have contributed (lower graph)

Preterm (<37weeks) unexplained still birth, deaths attributed to IUGR

There was a highly significant increase in PNM attributed to unexplained stillbirth $(P<0.01)$ and a highly significant decrease in PMR related to IUGR $(p<0.01)$ over the study period but the combined PNM was unchanged averaging $1 / 1000$ births. 
The incidence of overt placental abruption has remained the same over the study period, however the overall PMR attributed to placental abruption has fallen dramatically $(P<0.001)$; an improvement largely attributed to a significant fall in deaths before 37 week gestation while a non significant decrease was seen among those greater than
37 weeks $(\mathrm{P}=0.1)$.

Preterm delivery

The actual numbers of preterm births has increased almost two fold from a rate of 3.8 to $6.9 \%$ with in-utero transfers now accounting for half of babies less than 34 weeks gestation. The perinatal mortality calculated for deaths related to prematurity among births less than 37 weeks gestation has fallen from an average 50/1000 of in the eighties births to $12 / 1000$ births in recent years $(\mathrm{p}<0.001)$; due to an increased survival among the cohort < 30 weeks gestation.

Multiple pregnancy

The number of multiple pregnancies has increased significantly from a rate of $1 \%$ to $1.5 \%$ but the overall PMR is unchanged ranging from 0.4 to $0.7 / 1000$ births during the study period.

Rhesus Disease

There was a marked decline in deaths due to RCA at all gestations. In 1984 the PNMR from Rhesus disease was $0.65 / 1000$ and declined steadily throughout the 1990s. There have been no stillbirths or neonatal deaths since 1999 .

Post mortem rate

The post-mortem rate was close to $100 \%$ up to 1998 and then fell progressively to level out around $60 \%$ over the latter years of the study. When individual categories of cause of death were analysed, the rate of post-mortem was highest years of the study. When individual categories of cause of death were analysed, the rate of post-mortem was highest a classical placental abruption.

Figure 5: Trend for incidence of placental abruption and associated PNM/ 1000 deliveries (P< 0.001 )

\section{Discussion}

There has been a significant decline in the PNMR of normally formed births over the past twenty four years in Ireland. The sharpest decline occurred throughout the $1990 \mathrm{~s}$ while the reduction in PNMR appears to have plateaued over the last few years. The most striking reductions were seen in peripartum and post-term fetal deaths, deaths related to prematurity, deaths associated with placental abruption and the dramatic decline in deaths resulting from rhesus isoimmunisation. During this period, obstetric intervention in pregnancy has increased and NMH like many other units reports a continuing rise in caesarean section rates. Overall, during the course of this study, the CS rate at NMH has increased four fold. Although caution is required in interpreting a direct link between rising obstetric intervention such as induction of labour and caesarean section rate, the reduction in perinatal mortality has plateaued over the past few years while CS rates continue to rise steeply. This has led some authors to explore and question the role of increasing CS rate in the reduction of PNMR.

When individual causes of death are analysed, the most striking reductions in perinatal mortality occurred where a preventative strategy was available to address a specific cause of death. For example, the dramatic decline in deaths from Rhesus disease resulted from the introduction of rhesus immune prophylaxis to prevent isoimmunisation and the ability to identify and treat the anaemic fetus inutero. Similarly the reduction in perinatal death after 42 weeks may be reasonably attributed in part to the introduction in the late $1990 \mathrm{~s}$ of a policy of induction of labour at 42 weeks for postdates pregnancy and to improvements in fetal monitoring during labour. These changes also coincide with a very significant decline in intrapartum and early neonatal deaths attributable to asphyxia. There was a significant rise in the rate of unexplained stillbirths before 37 weeks with no change after 37 weeks. It has been argued that a decline in post-mortem rate may increase the rate of unexplained stillbirth but in this study unexplained SB had the highest post-mortem rate of $80 \%$. In one third of cases of unexplained SB, additional findings are reported such as subclinical placental abruption and cord accidents which are of uncertain significance. The lack of decline in unexplained SB is, gonsistent with previous work and is of particular concern as this group accounts for around half of all fetal deaths.

Data from England, Wales and Northern Ireland have shown a significant rise in SB rates from $2001^{12,13}$ and although this may relate to improved case ascertainment, it is likely that increasing maternal age $_{\text {a }}$ and a rising prevalence of obesity, hypertension and glucose intolerance are contributing to this increase. ${ }^{4-16}$ Certainly, this group warrants further study if we are to further reduce the PNMR. While the overall incidence of unexplained SB has increased, deaths related to IUGR have declined before 37 weeks and remain unchanged after 37 weeks. For the purpose of this study IUGR was diagnosed on the basis of post-mortem findings and not simply birth weight. It is conceivable that the reduction of deaths attributable to IUGR prior to term reflects improved antenatal surveillance and better timing of delivery. Certainly p, pstetric ultrasound and the use of Doppler flow studies has proved to be a useful tool in the management of IUGR. 1 is is unusual to allow pregnancies known to be growth restricted to proceed beyond term so one might speculate that deaths after 37 weeks relate to cases of missed IUGR.

It was interesting that although the incidence of placental abruption did not change significantly during the study period, the PNMR declined significantly. Further study of this population would be required to determine if this can be attributed to improved obstetric management or a reduction in the severity of abruption experienced. Many risk factors for placenta abruption are identified including hypertensive disorders, maternal age, prior abruption, low maternal weight, drug abuse, smoking, low socioeconomic group, chorioamnionitis, and multiple pregnancy. There has been a trend towards an increase in the incidence of placental abruption in the United States over the past two decades and while the reason for this is unclear and may be multifactorial, the trend is of concern and deserves further scrutiny.

It is impossible to draw meaningful conclusions from the superficial data on preterm deliveries within this study. The National Maternity Hospital has expanded its role as a tertiary referral centre over the course of this study and by 2007, almost one third (31.7\%) of deliveries less than 37 weeks were referred from other centres in Ireland and tended to be of lower gestation (< 30 weeks) and of complex case mix. Internationally many units report an increase in the rate of preterm delivery. In the USA, the majority of this increase has been attributed to a rise in late preterm deliveries ( 34 to 36 weeks) and to a rise in medically indicated preterm birth. In one Scottish study over 25 years, neonatal mortality improved in all subgroups of preterm birth regardless of obstetric antecedent of preterm. years, neonatal mortality improved in all subgroups of preterm birth regardless of obstetric antecedent of pretern mortality for medically induced but not spontaneous preterm births. However, in this study preterm birth still mortality for medically induced but not spontaneous preterm births. However, in this study preterm birth still ${ }^{3}$ accounted for two thirds of singleton stillbirths and two thirds of singleton neonatal deaths. PNMR can largely be attributed to improved neonatal care, a reduction in intrapartum and postterm fetal death, reduced perinatal mortality following placental abruption and better management of Rhesus disease. The lack of decline in unexplained stillbirth is an important finding and warrants further investigation. 


\section{References}

1. Bell R, Glinianaia SV, Rankin J, Wright C, Pearce MS, Parker L. Changing patterns of perinatal death, 1982-2000: a retrospective cohort study. Arch Dis Child Fetal Neonatal Ed 2004;89; F531-36.

2. Matthews TG, Crowley P, Chong A, McKenna P, MCGarvey C, ORegan M. Rising caesarean section rates: a cause for concern? BJOG. 2003;110:346-9.

3. Menacker F, Declercq E, Macdorman MF. Cesarean delivery: background, trends, and epidemiology. Semin Perinatol. $2006 ; 30 ; 235-41$

4. Meikle SF, Steiner CA, Zhang J, Lawrence WL. A national estimate of the elective primary cesarean delivery rate. Obstet Gynecol.2005;105:751-6.

5. Bell R, Parker L. MacPhail S, Wright C. Trends in the cause of late fetal death, 1982-2000 BJoG. 2004;111:1400-1407 6. Ahlenius I, Thomassen P. The changing panorama of late fetal death in Sweden between 1984 and 1991. Acta Obstet Gynecol Scand 1998; 78 : 408-414.

7. Pasupathy D, Wood AM, Pell JP, Fleming M, Smith GC. Rates of and factors associated with delivery-related perinatal death among term infants in Scotland. JAMA.2009;302:660-8.

8. Smith CS, Fretts RC. Stillbirth. The Lancet 2007; 370:1715-25

9. Aagaard-Tillery KM, Holmgren C, Lacoursiere DY, Houssain S, Bloebaum L Satterfield R, Branch DW, Varner MW. Factors associated with nonanomalous stillbirths: The Utah Stillbirth Database 1992-2002. Am J Obstet Gynecol 2006; 194:849-54. 10. Jonddottir G, Smarason AK, Geirsson RT, Bjarnadottir RI. No correlation between caesarean section rates and perinatal mortality of singleton infants over 2,500g. Acta obstet Gynecol Scand. 2009;88:621-3.

11. Cartlidge P. Dawson A. Stewart J, Vujanic G. Value and quality of perinatal and infant post-mortem examinations: cohort analysis of 400 consecutive deaths. BMJ 1995; 310:155-8.

12. CESDI. 8th Annual Report. London: maternal and Child health Research consortium, 2001.

13. Confidential enquiry into maternal and child health. Perinatal mortality surveillance, 2004 : England, Wales and Northern Ireland. London: CEMACH, 2006.

14. Huang D. Usher R. Kramer MS Yang H, Morin L, Fretts RC. Determinants of unexplained antepartum fetal deaths. Obstet Gynecol 2000 . 95 .

15. Mahony R. OHerlihy C. Thirty year trends in a large obstetric cohort. Ir Med J. 2003;96: 88-9.

15. Mahony R. OHerlihy C. Thirty year trends in a large obstetric cohort. Ir Med J. 2003; $96: 88-9$.
16. Nohr EA, Bech BH, Davies MJ, Frydenberg M, Henriksen TB, Olsen J. Prepregnancy obesity and fetal death: a study 16. Nohr EA, Bech BH, Davies MJ, Frydenberg M, Henriksen TB, Olsen J. Pr.
within the Danish National Birth cohort. Obstet Gynaecol 2005;106:250-9.

within the Danish National Birth Cohort. Obstet Gynaecol 2005; $106: 250-9$. 17. Baschat AA, Gembruch U, Reiss I, Gortner L, Weiner CP, Harman CR. Relationship between arte

and perinatal outcome in fetal growth restriction. Ultrasound Obstet Gynecol. $2000 ; 16: 407-13$. 18. Ghosh CS, Gudmundsson S. Uterine and umbilicai

19. Oyelese Y, Ananth CV. Placental abruption. Obstet Gynecol. 2006;108:1005-16

19. Oyelese Y, Ananth CV. Placental abruption. Obstet Gynecol. 2006;108:1005-16 20. Ananth CV, Oyelese $Y$, Yeo L, Predhan A, Vintzileos. Placenta abruption in the

temporal trends and potential determinants. Am J Obstet Gynecol. 2005;192:191-8. 21. Hofman C, Mahony R, Foley M, OHerlihy C, Gibbs R, Galan H, Lynch A. A Comparison of the incidence of pret

22. MC Intyre DD, Leveno K. Neonatal mortality and morbidity rates in late preterm births compared with births at 22. Mc Intyre DD, Leveno K. Neonatal

term. Obstet Gynecol. 2008; 111:35-41.
23. Norman J, Morris C, Chalmers J. The effect of changing patterns of obstetric care in Scotland (1980-2004) on rab 23. Norman J, Morris C, Chalmers J. The effect of changing patterns of obstetric care in Scotland (1980-2004) on
of preterm birth and its neonatal consequences: perinatal database study. PLos Med 2009; 6:e1000153. Epub 2009. 